\title{
lodine Intake is Associated with Thyroid Function in Mild to Moderately lodine Deficient Pregnant Women
}

\author{
Marianne Hope Abel, ${ }^{1,2,3}$ Tim I.M. Korevaar,, ${ }^{4,5}$ Iris Erlund, ${ }^{6}$ Gro Dehli Villanger,, Ida Henriette Caspersen, \\ Petra Arohonka, Jan Alexander, ${ }^{1}$ Helle Margrete Meltzer, ${ }^{1}$ and Anne Lise Brantsæter ${ }^{1}$
}

Background: Studies indicate that mild to moderate iodine deficiency in pregnancy may have a long-term negative impact on child neurodevelopment. These effects are likely mediated via changes in maternal thyroid function, since iodine is essential for the production of thyroid hormones. However, the impact of iodine availability on thyroid function during pregnancy and on thyroid function reference ranges are understudied. The aim of this study was to investigate the association between iodine intake and thyroid function during pregnancy.

Design: In a population-based pregnancy cohort including 2910 pregnant women participating in The Norwegian Mother and Child Cohort Study, we explored cross sectional associations of maternal iodine intake measured (1) by a food frequency questionnaire and (2) as iodine concentration in a spot urine sample, with plasma thyroid hormones and antibodies.

Results: Biological samples were collected in mean gestational week 18.5 (standard deviation 1.3) and diet was assessed in gestational week 22. Median iodine intake from food was $121 \mu \mathrm{g} /$ day (interquartile range 90, 160), and $40 \%$ reported use of iodine-containing supplements in pregnancy. Median urinary iodine concentration (UIC) was $59 \mu \mathrm{g} / \mathrm{L}$ among those who did not use supplements and $98 \mu \mathrm{g} / \mathrm{L}$ in the women reporting current use at the time of sampling, indicating mild to moderate iodine deficiency in both groups. Iodine intake as measured by the food frequency questionnaire was not associated with the outcome measures, while UIC was inversely associated with FT3 $(p=0.002)$ and FT4 $(p<0.001)$. Introduction of an iodine-containing supplement after gestational week 12 was associated with indications of lower thyroid hormone production (lower FT4, $p=0.027$, and nonsignificantly lower FT3, $p=0.17$ ). The 2.5th and 97.5th percentiles of TSH, FT4, and FT3 were not significantly different by groups defined by calculated iodine intake or by UIC.

Conclusion: The results indicate that mild to moderate iodine deficiency affect thyroid function in pregnancy. However, the differences were small, suggesting that normal reference ranges can be determined based on data also from mildly iodine deficient populations, but this needs to be further studied. Introducing an iodine-containing supplement might temporarily inhibit thyroid hormone production and/or release.

Keywords: iodine, pregnancy, thyroid function, dietary supplements, The Norwegian Mother and Child Cohort Study, MoBa

\section{Introduction}

$\mathbf{R}$ ESULTS FROM OBSERVATIONAL STUDIES, including the Norwegian Mother and Child Cohort Study (MoBa), have indicated that even mild to moderate iodine deficiency
(ID) in pregnancy might negatively affect child neurodevelopment (1-4). Iodine is an essential micronutrient, as it is an integral part of the thyroid hormones thyroxine (T4) and triiodothyronine (T3). Severe ID results in depleted iodine stores and a failure to sustain normal thyroid hormone levels

\footnotetext{
${ }^{1}$ Division of Infection Control and Environmental Health, Norwegian Institute of Public Health, Oslo, Norway.

${ }^{2}$ Department of Nursing and Health Promotion, Faculty of Health Sciences, OsloMet - Oslo Metropolitan University, Oslo, Norway.

${ }^{3}$ Department of Nutrition, Tine SA, Oslo, Norway.

${ }_{5}^{4}$ Department of Internal Medicine, Erasmus Medical Center, Rotterdam, The Netherlands.

${ }_{5}^{5}$ Academic Center for Thyroid Diseases, Erasmus Medical Center, Rotterdam, The Netherlands.

${ }^{6}$ Genomics and Biomarkers Unit, National Institute for Health and Welfare, Helsinki, Finland.

${ }^{7}$ Division of Mental and Physical Health, Norwegian Institute of Public Health, Oslo, Norway.
}

(C) Marianne Hope Abel et al., 2018; Published by Mary Ann Liebert, Inc. This Open Access article is distributed under the terms of the Creative Commons Attribution Noncommercial License (http://creativecommons.org/licenses/by-nc/4.0/) which permits any noncommercial use, distribution, and reproduction in any medium, provided the original author(s) and the source are cited. 
(5). The fetus is entirely dependent on an adequate and stable supply of these hormones, especially during the first half of pregnancy, until the fetal thyroid gland is developed. Thyroid hormones are essential in fetal growth, and particularly for cells in the central nervous system and for the structural and functional development of the brain (6). In areas with chronic moderate to severe ID, children score an estimated 8-10 points lower on IQ tests (7). Other dietary factors may contribute to aggravate symptoms of ID. Examples are deficiencies of other nutrients that are important for thyroid function (e.g., selenium and iron) (8), and intake of cruciferous vegetables that are known to inhibit iodine uptake and utilization by the thyroid due to high content of thiocyanate (9). Excessive iodine intake during pregnancy may also result in thyroid hypofunction (10). The association of iodine intake with thyroid function is U-shaped, and studies indicate that the range of optimal intake is narrow (11).

The recommended daily intake of iodine for pregnant women varies somewhat. The World Health Organization (WHO) recommends $250 \mu \mathrm{g} /$ day (or a population median urinary iodine concentration (UIC) $\geq 150 \mu \mathrm{g} / \mathrm{L}$ ), which is almost $70 \%$ higher than that of nonpregnant women, to compensate both for an increased turnover of iodine in pregnancy and depleted iodine stores in many populations (12). The European Food Safety Authority has a lower recommendation of $200 \mu \mathrm{g} /$ day but states that it is for populations with adequate iodine status prior to conception (13). The Nordic recommendation is even lower, at $175 \mu \mathrm{g} / \mathrm{day}$ (14), while in the United States, the recommended daily intake is $220 \mu \mathrm{g} / \mathrm{day}$ and the estimated average requirement is $160 \mu \mathrm{g} / \mathrm{day}(15,16)$. These recommendations are based only on limited data because the association of iodine intake with thyroid function during pregnancy has remained understudied. However, all the above-mentioned recommendations unanimously recommend $150 \mu \mathrm{g} /$ day for nonpregnant women (12-15).

During pregnancy, thyroid hormone production increases in order to meet the needs of the mother and fetus. Thus, thyroid hormone concentrations in pregnant women differ from nonpregnant women, and also change considerably throughout the gestation period (15). Other important determinants of measured hormone levels are body mass index (BMI), ethnicity, smoking habits, thyroid autoimmunity, and method of analysis, especially for free thyroid hormones (17). The 2017 guidelines of the American Thyroid Association (ATA) recommend using population- and assay-specific reference ranges for the thyroid hormones in defining diagnostic criteria for thyroid disorders and to avoid basing reference values on ID populations (15). However, there is a lack of knowledge regarding the potential impact of mild to moderate ID on thyroid hormone reference ranges (i.e., the $2.5-97.5 \%$ range) (17).

According to the World Health Organization (18), iodine supplements should be recommended for pregnant women in areas of inadequate iodine intake. Although the majority of US pregnant women have a sufficient iodine intake, the ATA recommends that all pregnant and lactating women in the United States use iodine supplements (19). Yet, there is uncertainty regarding the benefit and safety of recommending iodine supplements for pregnant women in areas with mild to moderate ID (20). In severe ID, iodine supplementation reduces the risk of thyroid hypofunction, but in mild to moderate ID, results from studies are not consistent (5). Some studies indicate that iodine supplementation may result in a temporary "stunning effect" of the thyroid gland with a transient lower thyroid hormone production $(21,22)$. Other studies find that supplements may be beneficial or have no effect on thyroid function or child outcomes $(20,23-25)$. In $\mathrm{MoBa}$, maternal iodine supplement use initiated in pregnancy was related to increased risk of child behavior problems and $\operatorname{ADHD}(3,4)$, but not to language or motor development $(3)$.

In a population of pregnant women from a mild to moderately ID population we aimed to

1. Study the associations of iodine intake measured by (i) a food frequency questionnaire (FFQ) and (ii) by urinary iodine concentration (UIC), with biomarkers of thyroid function;

2. Define thyroid function reference ranges for thyrotropin (TSH), FT4, and FT3 and investigate whether these differ according to iodine intake; and

3. Investigate the impact of current iodine supplement use, and also of timing of initiation, on thyroid function and autoimmunity.

\section{Subjects and Methods}

\section{Subjects and design}

This study is embedded within MoBa eTox, a substudy of the prospective population-based pregnancy cohort MoBa conducted by the Norwegian Institute of Public Health (26). Pregnant women in their first trimester were recruited from all over Norway during the years 1999 to 2008 and asked to answer questionnaires (in Norwegian) at regular intervals during pregnancy and after delivery. More than $99 \%$ of participants are of Caucasian origin. Pregnancy and birth records from the Medical Birth Registry of Norway are linked to the MoBa database (27). The women consented to participation in $41 \%$ of the pregnancies. The cohort now includes 114,500 children, 95,200 mothers, and 75,200 fathers. The current study is based on version 10 of the qualityassured data files released for research in 2017.

The participants in MoBa eTox $(n=2999)$ is a selected sample of MoBa consisting of participants who had responded to all questionnaires in MoBa up to child's age 3 years and had delivered all biological samples in mid pregnancy and at birth. Only singleton pregnancies were included. Children with autism, suspected autism, or symptoms of severe language delay were reserved for another substudy and those mother-child pairs were thus not included in MoBa eTox.

For our study ( $n=2910)$, we additionally excluded women reporting use of thyroid medication in pregnancy (gestational week $0-24)$, and women with calculated energy intakes $<4.5$ MJ or $>20$ MJ or more than 3 blank pages in the FFQ (28) (see flowchart of inclusion in Fig. 1).

\section{Exposure variables - iodine intake and UIC}

The MoBa FFQ was specifically designed for the MoBa study and in use from 2002 (29). It was completed by participating women around gestational week (GW) 22 , and is a semi-quantitative questionnaire designed to capture dietary habits and use of dietary supplements during the first half of pregnancy. It included questions about intake of 255 food 


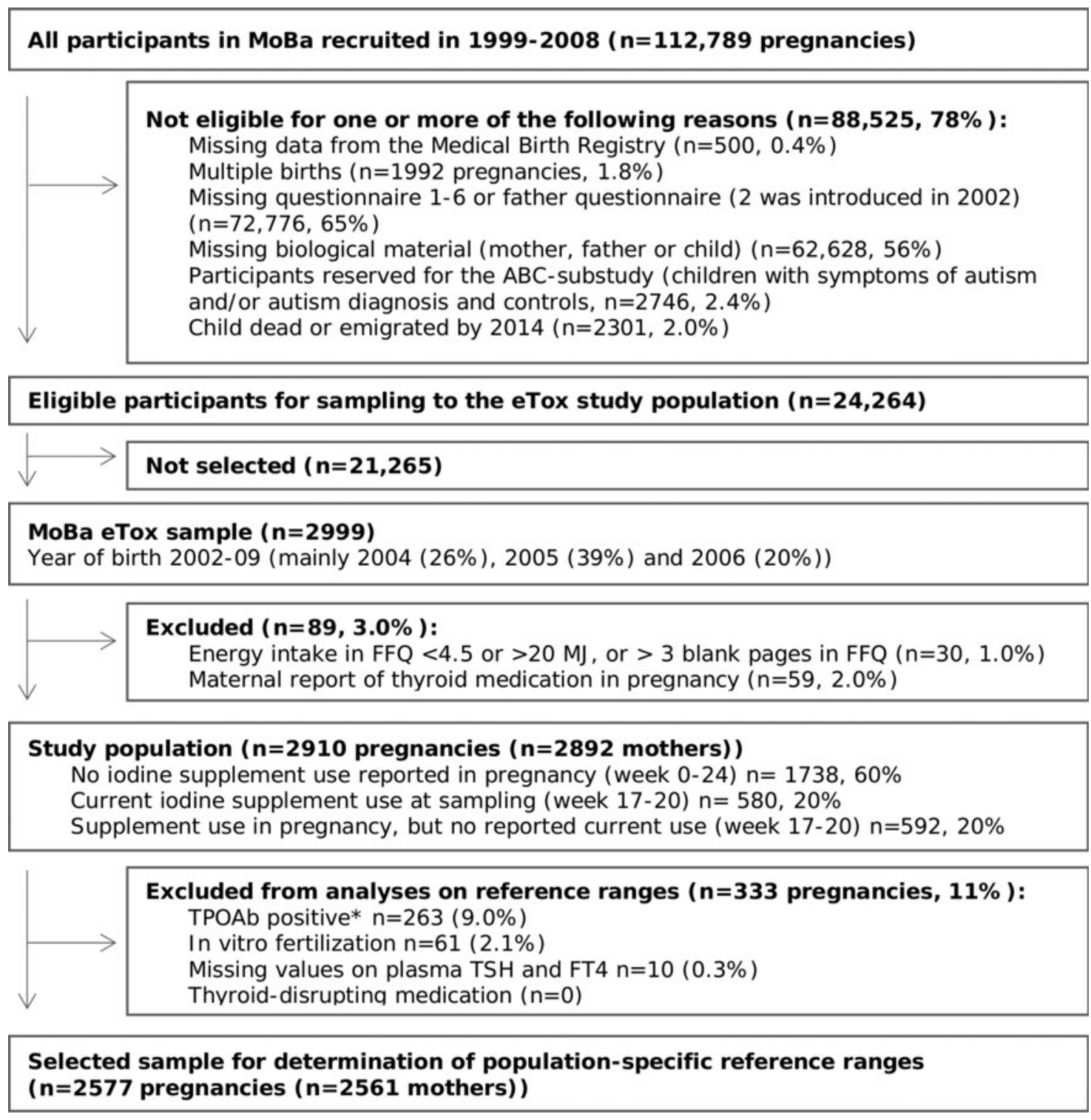

FIG. 1. Flow chart of inclusion. *TPOAb positive according to manufacturer-defined cutoff for nonpregnant women (TPOAb >4.11 IU/mL). FFQ, food frequency questionnaire; FT4, free thyroxine; MoBa, Norwegian Mother and Child Cohort Study; TPOAb, thyroid peroxidase antibodies; TSH, thyrotropin.

items or dishes (28). Intake of specific foods and nutrients were calculated based on standard Norwegian portion sizes, the Norwegian food composition table, analysis of Norwegian food samples $(30,31)$, and data on the content of more than 1000 dietary supplements collected from suppliers (32).

As reported previously (3), the MoBa FFQ was shown to be a valid tool for ranking pregnant women according to high and low intakes of energy, nutrients, and foods (33). Milk and seafood are the main dietary sources of iodine in Norway; thus, iodine intake is highly variable depending on individual food choices (34). Iodine was validated separately and iodine intake by the FFQ, including supplemental iodine, showed good agreement with the reference methods ( $24 \mathrm{~h}$ urinary iodine excretion and 4 days weighed food diary; triangular validity coefficient for total iodine intake by the FFQ was 0.62 [95\% confidence interval $0.46,0.77])(35,36)$.
Timing of iodine intake from supplements was reported in the general questionnaires one (GW 17), and three (GW 30). Supplement use was coded in three categories (no use in pregnancy, current use in GW 17-20, and use reported in pregnancy but not in GW 17-20). Timing of first report of iodine-containing supplements was coded in four categories (never, week 0-26 before pregnancy, GW 0-12, and GW 13-20).

Blood and urine samples were collected at the routine ultrasound examination offered in GW 18 (37). UIC was explored both as a crude measure $(\mu \mathrm{g} / \mathrm{L})$ and adjusted for hydration status by the "residual method" (38) (i.e., residuals after regressing log UIC on log urinary creatinine plus predicted UIC at the median creatinine concentration, obtaining UIC adjusted for creatinine [UIC $\sim \mathrm{Cr}$ ]). The method is frequently used in nutritional epidemiology to control for energy-intake in dietary surveys and has also been reported 
for UIC adjustment (39). UIC $\sim \mathrm{Cr}$ represents an estimate of UIC at a median hydration state.

\section{Outcome variables: Thyroid function biomarkers}

TSH, free T4 (FT4), free T3 (FT3), thyroid peroxidase antibodies (TPOAb), and thyroglobulin antibodies (TgAb) were measured in plasma samples from mid pregnancy (mean gestational week 18.5, standard deviation 1.3). For TPOAb and $\mathrm{TgAb}$, the 92nd percentile was chosen as a cutoff for defining antibody positivity based on previous literature (40) and on exploring associations between measured antibody concentrations and TSH and FT4 concentrations in our study sample (Supplementary Figs. S1 and S2; Supplementary Data are available online at www.liebertpub.com/thy). For TSH, FT4, and FT3, the cutoffs for defining low and high values were set to the 2.5 th and 97.5 th percentiles in a subsample of TPOAb negative women after exclusion of women that underwent in vitro fertilization (IVF).

\section{Analytical procedures}

Samples of urine and blood were collected at the 50 participating hospitals, and samples were shipped by ordinary mail (unrefrigerated shipment) in a vacutainer for long-term freezing at a central biorepository (37). Storage temperature was $-80^{\circ} \mathrm{C}\left(-20^{\circ} \mathrm{C}\right.$ for whole blood $)$.

UIC was determined at the National Institute for Health and Welfare (THL) in Helsinki (Finland) by inductively coupled plasma-mass spectrometry (ICP-MS) using an Agilent 7800 ICP-MS system (Agilent Technologies Inc., Santa Clara, CA). In brief, $100 \mu \mathrm{L}$ of urine sample was extracted using ammonium hydroxide solution. Tellurium was used as an internal standard. On the ICP-MS, m/z = 127 was scanned for iodine determination. The limit of quantification of the method was $2 \mu \mathrm{g} / \mathrm{L}$ and the linearity was $t(r=0.9999$ up to $1500 \mu \mathrm{g} / \mathrm{L}$ ). The National Institute of Standards and Technology standard reference materials SRM2670a (with certified mass concentration value) and SRM3668 Level 1 and Level 2 were used to validate the method. The coefficient of variation (CV) of control samples was 3.0-4.8\%. The laboratory at THL participated in the Ensuring the Quality of Urinary Iodine Procedures Program organized by the Centers for Disease Control and Prevention three times per year.

Urinary creatinine was analyzed by an enzymatic method using Multigent Creatinine (Enzymatic) Assay (SENTINEL CH. Sp.A.) using the ARCHITECT ${ }^{\circledR}$ c System (Abbott Laboratories, Abbott Park, IL) at THL. CV of control samples was $1.4-1.7 \%$. The laboratory participated in an external quality assessment scheme for urinary creatinine organized by Labquality (Finland).

Plasma TSH, FT3, FT4, TPOAb, and TgAb concentrations were analyzed at THL using chemiluminescent microparticle immunoassays. Reagents and equipment (Architect ci8200 analyzer) were from Abbott Laboratories. CV of control samples (Bio-Rad Liquichek Immunoassay Plus lot 40860 high and low level, and in-house control, $n=181$ ) were as follows: TSH 1.8-2.9\%, FT3 2.5-4.7\%, FT4 2.7-8.5\%, TPOAb $3.7-5.2 \%$, TgAb 4.1-4.4\%. The laboratory participated in external quality assessment schemes for thyroid hormones and antibodies organized by Labquality (Finland).

Plasma ferritin was analyzed at THL using a chemiluminescent microparticle immunoassay. The coefficient of var- iation of control samples (Bio-Rad Liquichek immunoassay Plus lot 40860 high and low level, and in-house control, $n=181$ ) were $2.7-3.7 \%$. The laboratory participated in an external quality assessment scheme for ferritin organized by Labquality (Finland).

Whole blood selenium was analyzed at Lund University in Sweden by inductive coupled plasma mass spectrometry (ICP-MS; iCAP Q, Thermo Fisher Scientific, Bremen, $\mathrm{GmbH}$ ) equipped with collision cell with kinetic energy discrimination and helium as collision gas. The detection limit was $3.2 \mu \mathrm{g} / \mathrm{L}$ and the coefficient of variation was $1.5 \%$. The analytical accuracy was verified towards certified reference material; Seronorm Trace elements whole blood L-1 and L-2 (SERO AS, Billingstad, Norway).

\section{Covariates}

Data on covariates were obtained from different sources: Maternal age at delivery was obtained from the Medical Birth Registry of Norway. Maternally reported prepregnancy body weight and height for the calculation of body mass index (BMI), maternal education ( $\leq 12,13-16, \geq 17$ years), parity (previous pregnancies $\geq 22$ weeks: $0,1, \geq 2$ ), maternal chronic illness (asthma, diabetes, inflammatory bowel disease, rheumatic disease, epilepsy, multiple sclerosis or cancer before or during pregnancy [yes/no]), and smoking in pregnancy (average cigarettes/day GW 0-17) was included from questionnaire 1. Energy intake, fiber intake (as a marker of a healthy dietary pattern), and intake of cruciferous vegetables (high in glucosinolates, potent goitrogens) were calculated based on the FFQ.

Selenium status (whole blood selenium $<80, \geq 80 \mu \mathrm{g} / \mathrm{L}$ ), and iron status (plasma ferritin $<12,12-29.9, \geq 30 \mu \mathrm{g} / \mathrm{L}$ ) were measured in the blood samples in mid-pregnancy, and creatinine concentration was measured in the urine samples. Gestational age at blood and urine sampling was estimated based on the ultrasound examination, which took place the same day as the sampling and, if missing, on the first day of last menstruation.

\section{Ethics}

$\mathrm{MoBa}$ is conducted according to the guidelines laid down in the Declaration of Helsinki and written informed consent was obtained from all participants. MoBa has obtained a license from the Norwegian Data Inspectorate. The current study was approved by The Regional Committee for Medical Research Ethics South East Norway 2014/2211.

\section{Statistics}

Statistical analyses were performed in STATA (version 15.0; Stata Corp., College Station, TX). The package postrcspline for STATA was used for graphing of flexible models (41).

In total, $4.8 \%$ of the women had missing values on one or more of the covariates: pre-pregnancy BMI $(n=54$, $1.9 \%)$, maternal education $(n=62,2.1 \%)$, gestational age at sampling $(n=10,0.3 \%)$, and whole blood selenium $(n=18$, $0.6 \%)$. Missing values in covariates were imputed using multiple imputation by chained equations in STATA, and 20 imputed datasets were generated for analyses.

Associations between continuous exposures and outcomes were modelled flexibly by use of restricted cubic splines 
(three knots). Covariates were included in the models based on previous knowledge and a directed acyclic graph (Supplementary Fig. S3).

Linear regression was used to model associations with continuous outcomes. To obtain close to normal distribution of the outcomes, we used the square root of TSH, and we excluded the highest percentile of FT4 and FT3. Logistic regressions were used to model the odds of antibody positivity. Adjusted models included the following covariates: maternal age, education, parity, pre-pregnancy BMI, fiber intake, smoking in pregnancy, plasma ferritin, whole blood selenium, and gestational age at sampling. Urinary creatinine was included in models with $\mathrm{UIC} \sim \mathrm{Cr}$ as exposure, energy intake in models with iodine from food as exposure, and IVF in models with supplement use as exposure (supplement use was more commonly reported in IVF pregnancies).

Possible interaction effects were explored for iodine supplement use, dichotomous variables of whole blood selenium $(<80 \mu \mathrm{g} / \mathrm{L})$ and plasma ferritin $(<20 \mu \mathrm{g} / \mathrm{L})$, intake of cruciferous vegetables $(>75$ th percentile), and smoking in pregnancy (yes/no). The potential interactions were explored by including an interaction term with iodine from food in the spline models.

$p$-Values are reported for overall associations between continuous exposures and outcomes by testing the coefficients of all spline transformations equal to zero. The tests for nonlinearity were performed by testing the coefficient of the second spline transformations equal to zero. All statistical tests were performed on the imputed datasets $(n=20)$ and adjusted for random effects of person clusters since some women participated with more than one pregnancy $(n=9)$. Results are reported including robust $95 \%$ confidence intervals. A $p$-value $<0.05$ was considered statistically significant. Sensitivity analyses included repeating analyses on thyroid hormones and TSH, but (1) excluding antibody positive women, or (2) including the upper first percentile for outcomes FT3 and FT4.

To explore potential effects of iodine status on populationspecific reference ranges for TSH and thyroid hormones, we used quantile regression regressing the 2.5th and 97.5th percentile by categories of UIC, UIC $\sim \mathrm{Cr}$, and by categories of iodine intake (restricted to nonusers of iodine supplements) in participants who were not TPOAb-positive ( $>4.11$ IU/mL, cutoff suggested by manufacturer) and not IVF treated.

\section{Results}

Characteristics of the study population are shown in Table 1. A more detailed overview of population characteristics by exposure level is provided in Supplementary Table S1.

\section{lodine intake and UIC}

Median habitual iodine intake from food based on the FFQ was $121 \mu \mathrm{g} /$ day. Four percent had a calculated iodine intake from food reaching the WHO recommended intake of $250 \mu \mathrm{g} /$ day for pregnant women, and $30 \%$ reaching the recommended intake of $150 \mu \mathrm{g} /$ day for nonpregnant women. The low intake was reflected in a low median UIC in supplement nonusers ( $59 \mu \mathrm{g} / \mathrm{L}, n=1738)$. In this subgroup, iodine from food according to the FFQ correlated weakly with
Table 1. Descriptive Characteristics of the Study Population ( $N=2910$ PREgnancies)

\begin{tabular}{lc}
\hline Study sample/pregnancies, $n$ & 2910 \\
Maternal age at delivery (years), & $30.3(4.2)$ \\
mean (SD) &
\end{tabular}

Gestational age at sampling

(weeks), mean (SD)

Prepregnancy BMI $\left(\mathrm{kg} / \mathrm{m}^{2}\right)$, mean (SD)

Parity, \%

0

52

$1 \quad 33$

2 or more $\quad 14$

Maternal education, \%

$\leq 12$ years

13-16 years

$>16$ years

Other/missing

Married/cohabitant, \%

In vitro fertilization, $\%$

Smoking in pregnancy, $\%$

Occasionally

Daily

Chronic illness, \%

Household income, \%

Low

Medium

High

Missing

Iodine from food ( $\mu \mathrm{g} /$ day $)$, median (IQR)

UIC $(\mu \mathrm{g} / \mathrm{L})$, median (IQR)

Urinary creatinine $(\mathrm{g} / \mathrm{L})$, median (90\% range)

UIC $(\mu \mathrm{g} / \mathrm{g}$ creatinine), median (IQR)

$\mathrm{UIC} \sim \mathrm{Cr}(\mu \mathrm{g} / \mathrm{L})$, median $(\mathrm{IQR})$

UIC, \%

$\geq 150 \mu \mathrm{g} / \mathrm{L}$ ( sufficient $^{\mathrm{a}}$ )

$0-150 \mu \mathrm{g} / \mathrm{L}$ (insufficient $^{\mathrm{a}}$ )

$0-100 \mu \mathrm{g} / \mathrm{L}$

$0-50 \mu \mathrm{g} / \mathrm{L}$

Plasma FT4 [pmol/L], median [95\% range]

Plasma FT3 [pmol/L], median [95\% range]

Plasma TSH [mU/L], median [95\% range]

Plasma TPOAb positive, ${ }^{\mathrm{b}} \%$

Plasma TgAb positive, ${ }^{\mathrm{b}} \%$

Plasma ferritin (ng/mL), median (IQR)

Empty iron stores (P-Fe $<12$ ng/mL), \%

Low iron stores (P-Fe 12-29.9 ng/mL), \%

Whole blood selenium $(\mu \mathrm{g} / \mathrm{L})$, median (IQR)

Low selenium $(<80 \mu \mathrm{g} / \mathrm{L}), \%$

\section{6}

47

25

2.1

98.4

2.2

14

3.8

8.9

26

43

$121(90,160)$

$68(35,116)$

$0.76(0.17,1.92)$

$91(61,139)$

$74(55,105)$

14
86
68
37
$12.6[10.3,15.7]$

$4.9[4.0,6.0]$

$1.2[0.4,2.9]$

8.1

7.9

$33(20,56)$

9.0

35

$102(89,117)$

${ }^{\mathrm{a}}$ Population median UIC $<150 \mu \mathrm{g} / \mathrm{L}$ is the recommended cutoff for defining inadequate iodine intake in pregnancy by the World Health Organization.

${ }^{\mathrm{b}}$ Antibody positivity was defined as values above the 92nd percentile ( $>6.6 \mathrm{IU} / \mathrm{mL}$ for TPOAb and $>7 \mathrm{IU} / \mathrm{mL}$ for $\mathrm{TgAb}$ ).

$\mathrm{IQR}$, interquartile range; $\mathrm{SD}$, standard deviation; $\mathrm{TgAb}$; thyroglobulin antibodies; TPOAb, thyroid peroxidase antibodies; UIC, urinary iodine concentration; UIC $\sim \mathrm{Cr}$, UIC adjusted for creatinine. 
spot UIC measured in $\mu \mathrm{g} / \mathrm{L}$ (Spearman correlation $r=0.20$, $p<0.001$ ), with UIC measured in $\mu \mathrm{g} / \mathrm{g}$ creatinine (Spearman $r=0.30, p<0.001$ ), and with UIC adjusted for creatinine by the residual method (UIC $\sim \mathrm{Cr}$ ) (Spearman $r=0.28, p<0.001$ ). The association between iodine from food and UIC in nonusers of iodine supplements is shown in Figure 2. Habitual iodine intake explained $3.9 \%$ of the variation in UIC $(p<0.001)$ when regressing log iodine from food on log UIC.

The UIC differed according to reported use of iodinecontaining supplements (Supplementary Fig. S4). In participants reporting supplement use at the time of sampling (GW 17-20, $n=580$ ) median UIC was $98 \mu \mathrm{g} / \mathrm{L}$, significantly higher than in those not using supplements (median $59 \mu \mathrm{g} / \mathrm{L}, p<0.001$ ). There was no data available on the dosage of current intake of iodine from supplements, but the most frequently reported supplements contained $\sim 150 \mu \mathrm{g}$ per recommended daily dose.

\section{lodine intake and thyroid function and autoimmunity}

Habitual iodine intake from food calculated by the FFQ was not significantly associated with any of the outcome measures (Figs. 3 and 4 and Supplementary Fig. S5), but UIC was inversely associated with FT4 and FT3 (Fig. 3). UIC $\sim \mathrm{Cr}$ lower than roughly $100 \mu \mathrm{g} / \mathrm{L}$ was associated with an increase in mean FT3. Excluding thyroid antibody positive women (TPOAb and/or TgAb >92nd percentiles) did not change the results (Supplementary Fig. S6). Results from crude models are included in Supplementary Figures 7 and 8.

The results did not differ when taking into account iron status, selenium status, or smoking during pregnancy. Only smoking during pregnancy was associated with a lower FT4 and higher FT3 (both $p<0.001$ ). Intake of cruciferous vegetables was generally low (median: $18 \mathrm{~g} /$ day, 90 percent range: $2-56 \mathrm{~g} /$ day) and was not associated with the thyroid function parameters.

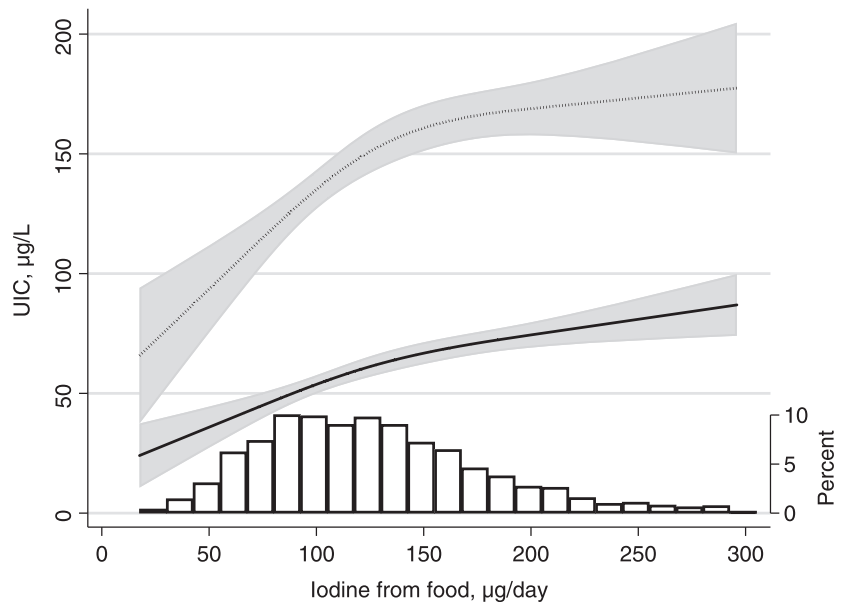

FIG. 2. Association between iodine from food (measured by the food frequency questionnaire covering gestational weeks 0-22) and spot UIC measured at mean gestational week 18.5 (SD 1.2) in nonusers of iodine supplements $(n=1738)$. The solid line represents the estimated median, the dotted line shows the 90th percentile, and shaded areas are the $95 \%$ confidence intervals. The predicted values were estimated by quantile regression adjusting for calculated energy intake. The histogram illustrates the distribution of iodine intake from food. UIC, urinary iodine concentration.
Population-specific reference ranges for TSH, FT4, and FT3

Table 2 shows the 2.5 th and 97.5 th percentiles for plasma TSH, FT4, and FT3 after excluding TPOAb-positive and IVF pregnancies. There was no evidence of any association between either iodine intake or UIC and reference ranges of plasma TSH, FT4 or FT3 (Table 3 and Supplementary Table S2). However, even the groups with the highest iodine intakes had inadequate iodine intake according to the WHO criteria (i.e., median UIC $<150 \mu \mathrm{g} / \mathrm{L}$ ). Additionally excluding remaining $\operatorname{TgAb}$-positive women $(n=147)$ did not change the results (Table 2 ).

Based on the reference ranges determined in this subgroup (listed in Table 2), 3.2\% of the total study population had subclinical hypothyroidism (high TSH and normal FT4), $0.1 \%$ had overt hypothyroidism (high TSH and low FT4), 2.3\% had isolated hypothyroxinemia (low FT4 and normal TSH), $2.3 \%$ had subclinical hyperthyroidism (low TSH and normal FT4), and $0.1 \%$ had overt hyperthyroidism (low TSH and high FT4 or FT3). The prevalence of thyroid antibody positivity was $11.4 \%$ by our population-based cutoffs at $>92$ nd percentile (i.e., TPOAb $>6.6 \mathrm{IU} / \mathrm{mL}$ or $\operatorname{TgAb}>7 \mathrm{IU} / \mathrm{mL})$.

\section{lodine from supplements and thyroid function}

Forty percent reported use of iodine-containing supplements in gestational week $0-24$, and $20 \%$ reported use at the time of sampling (GW 17-20). Current iodine supplement use (yes/no) was not significantly associated with the outcomes.

Information on timing of iodine supplement use was specified by $74 \%$ of the users of iodine-containing supplements in pregnancy. Of these, $40 \%(n=347)$ reported first use in weeks $1-26$ before conception, $37 \%(n=323)$ in first trimester, and $22 \%(n=194)$ in gestational weeks 13-20. Measures of thyroid parameters by timing of supplement use are shown in Figure 5 and Supplementary Figure S9. A recent initiation of supplement use (after GW 12) was associated with a lower mean FT4 (beta $=-0.21, p=0.027$ ), and also lower FT3 and higher TSH, but not statistically significant (beta $-0.05, p=0.17$ and beta $0.02, p=0.27$ ) compared with nonuse of supplements. There was also a tendency of a higher risk of hypothyroxinemia (i.e., plasma FT4 $<10.3 \mathrm{pmol} / \mathrm{L}$, the population specific reference range) (odds ratio 2.09, $p=0.089$ ). Results from crude models are included in Supplementary Figure S10.

\section{Discussion}

In this mildly to moderately iodine deficient pregnant population, we show that a higher UIC was associated with a slightly lowered FT4 concentration and that a low UIC, from a cutoff point of roughly $100 \mu \mathrm{g} / \mathrm{L}$, was associated with an elevated FT3. Furthermore, we show that reference ranges for TSH, FT4 or FT3 did not differ by UIC or by calculated habitual iodine intake. A recent introduction of an iodine containing supplement was associated with a lower thyroid function, whereas more long-term use was not (i.e., use initiated before conception or early in the pregnancy).

\section{Calculated iodine intake and UIC}

Calculated habitual iodine intake from food in nonusers of supplements explained only $4 \%$ of the variation in UIC, 

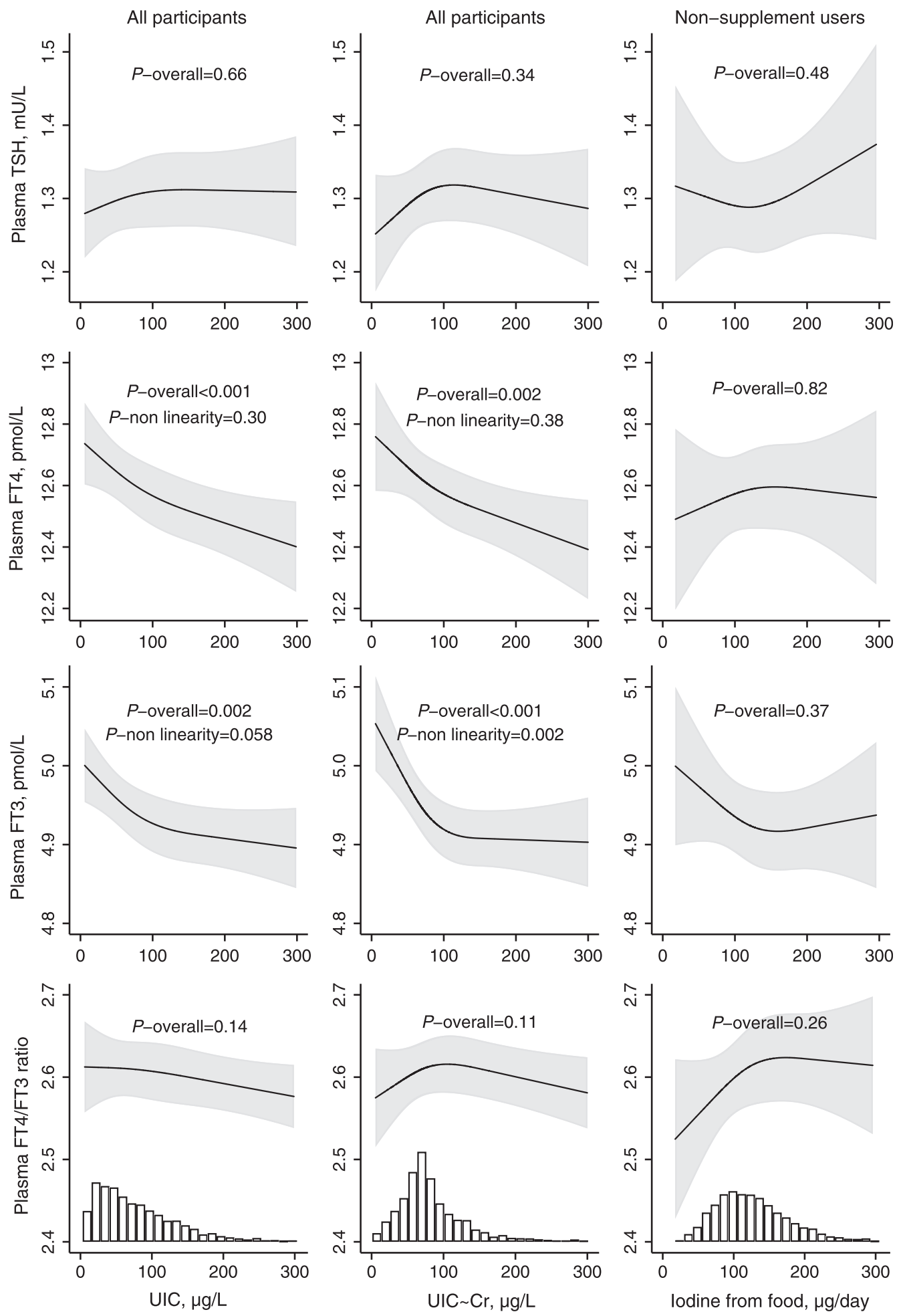

FIG. 3. Adjusted associations between UIC (column $1 ; n=2900$ ), creatinine-adjusted UIC (column $2 ; n=2900$ ), and iodine from food (column 3, restricted to nonusers of iodine supplement; $n=1730$ ) and TSH, FT4, and FT3. For TSH, the geometric mean was modelled (by square root transformation), and for FT3 and FT4, the highest first percentile was excluded to obtain $\sim$ normal distribution. Including the upper first percentile of FT3 and FT4 did not change the results (Supplementary Fig. S5). FT3, free triiodothyronine.

which highlights the restricted value of a spot UIC as a measure of habitual iodine intake at an individual level. However, both the median UIC and the variance in UIC increased with calculated habitual iodine intake (Fig. 2), affirming that the MoBa FFQ provides a valid measure of the habitual iodine intake as reported previously (36). The increase in median UIC started to level off at about $150 \mu \mathrm{g} / \mathrm{L}$ (equivalent to the 70 th percentile), indicating overreporting of food intake at higher levels. Median UIC remained below $100 \mu \mathrm{g} / \mathrm{L}$ over the whole range of calculated iodine intakes by the FFQ, thus the whole 

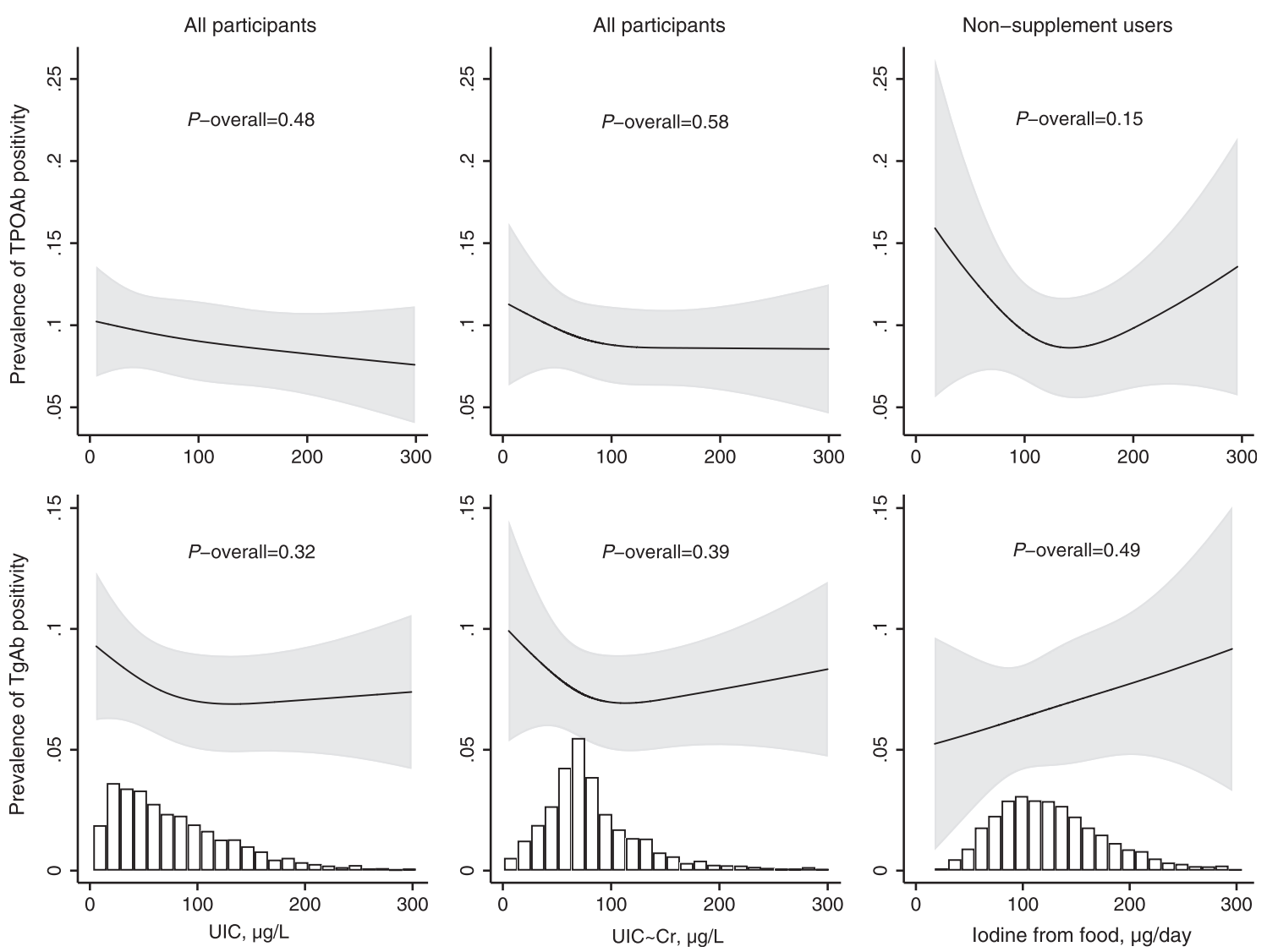

FIG. 4. Prevalence of thyroid antibody positivity by UIC (column $1 ; n=2900$ ), creatinine-adjusted UIC (column 2; $n=2900$ ), and by iodine from food (column 2, restricted to nonusers of iodine supplements; $n=1730$ ), adjusted models. Antibody positivity was defined as antibody plasma concentration $>92$ nd percentile.

group of supplement nonusers in this study had an insufficient habitual iodine intake in pregnancy (i.e., population median $\mathrm{UIC}<150 \mu \mathrm{g} / \mathrm{L})$. Even the women reporting current use of iodine-containing supplements had median UIC $<100 \mu \mathrm{g} / \mathrm{L}$. Explanations for the low UIC in iodine supplement users might be that the supplements were not consumed daily by all users, or that supplemental iodine was trapped in the thyroid to refill depleted iodine stores. The latter was indicated in a recent study in Bangladesh in pregnant women with comparable median UIC as in our supplement nonusers (42). Introducing a supplement containing $250 \mu \mathrm{g}$ iodine/day did not result in an increase in UIC.

\section{lodine intake and thyroid function}

In ID, a range of effective autoregulatory mechanisms are triggered in the thyroid and other tissues to maintain euthyroidism. This involves a preferential secretion of T3 over $\mathrm{T} 4$, whereas an elevated TSH is rarely seen in populations with mild to moderate ID (43). In our study, we did observe a higher FT3 when habitual iodine intake from food was below $\sim 150 \mu \mathrm{g} /$ day (reflected in a UIC below $\sim 100 \mu \mathrm{g} / \mathrm{L}$ ), but the trends in the FT4/FT3 ratio did not reach statistical significance.

Our findings indicate that short-term iodine availability (measured by UIC) might have a partially different impact on thyroid hormones compared to long-term iodine intake (calculated by the FFQ). For FT3, our findings, both for UIC and for habitual iodine intake from food, indicate that a low iodine intake (below $\sim 150 \mu \mathrm{g} /$ day) is associated with a higher plasma FT3 (Fig. 3). This is in line with observations in both animal and human studies and reflects the autoregulatory mechanisms to preserve iodine in response to decreased availability of iodine (44). A preferential production of T3 to T4 saves one iodine atom per hormone produced, and at the same time secures availability of most maternal tissues to T3, the active form of thyroid hormone. However, the fetal brain may be vulnerable to lower maternal $\mathrm{T} 4$ since $\mathrm{T} 3$ in the fetal brain is predominantly generated locally from maternal T4 during early pregnancy (44). Several studies show that maternal hypothyroxinemia may result in suboptimal fetal brain development, and that this may particularly be the case before the onset of fetal thyroid hormone production at mid pregnancy $(45,46)$.

We observed that UIC was inversely associated with FT4, which is opposite to what one might expect, and also opposite to what was indicated for iodine intake by the FFQ (Fig. 3). We hypothesize that this finding might reflect that thyroid hormone production is inhibited during more acute higher iodine availability during pregnancy via a mechanism similar to that of the overt Wolff-Chaikoff effect. Interestingly, these associations were already present within the range of what is considered optimal and safe iodine intake. These results could indicate that pregnant women with mild to moderate ID who increase their iodine intake during pregnancy are more prone to having a (temporary) low FT4. This is supported by 
Table 2. The 2.5Th and 97.5th Percentiles (Reference Range) of TSH, FT4, and FT3 in TPOAB Negative Pregnant Women

\begin{tabular}{|c|c|c|}
\hline & Reference population $^{\mathrm{a}}$ & Also excluding $T g A b$-positive \\
\hline \multicolumn{3}{|l|}{ Plasma TSH, mU/L } \\
\hline$n$ & 2577 & 2430 \\
\hline 2.5th percentile $[95 \% \mathrm{CI}]$ & $0.39[0.36,0.44]$ & $0.41[0.36,0.45]$ \\
\hline Median $[95 \% \mathrm{CI}]$ & $1.19[1.17,1.22]$ & $1.19[1.16,1.22]$ \\
\hline 97.5th percentile $[95 \% \mathrm{CI}]$ & $2.70[2.62,2.80]$ & $2.70[2.60,2.79]$ \\
\hline \multicolumn{3}{|l|}{ Plasma FT4, pmol/L } \\
\hline$n$ & 2576 & 2429 \\
\hline 2.5th percentile $[95 \% \mathrm{CI}]$ & $10.3[10.2,10.4]$ & $10.3[10.2,10.4]$ \\
\hline Median $[95 \% \mathrm{CI}]$ & $12.6[12.5,12.6]$ & $12.5[12.5,12.6]$ \\
\hline 97.5th percentile $[95 \% \mathrm{CI}]$ & $15.6[15.3,16.0]$ & $15.6[15.3,16.0]$ \\
\hline \multicolumn{3}{|l|}{ Plasma FT3, pmol/L } \\
\hline$n$ & 2577 & 2430 \\
\hline 2.5 th percentile $[95 \% \mathrm{CI}]$ & $4.00[3.97,4.03]$ & $4.00[3.97,4.03]$ \\
\hline Median $[95 \% \mathrm{CI}]$ & $4.90[4.87,4.92]$ & $4.90[4.87,4.93]$ \\
\hline 97.5th percentile $[95 \% \mathrm{CI}]$ & $6.00[5.91,6.10]$ & $6.00[5.91,6.10]$ \\
\hline
\end{tabular}

Mean gestational week, 18.5; SD, 1.3 .

${ }^{a}$ Population characteristics: singleton pregnancies, not TPOAb positive (i.e., TPOAb $<4.11 \mathrm{IU} / \mathrm{mL}$ ), not current user of thyroid medication or thyroid disrupting medication, and not in vitro fertilization.

${ }^{\mathrm{b}}$ Additionally excluding TgAb positive ( $>4.11 \mathrm{IU} / \mathrm{mL}$ according to manufacturer cutoff $), n=147$.

FT3, free triiodothyronine; FT4, free thyroxine; TSH, thyrotropin.

our finding that a recent introduction of iodine-containing supplements (GW 13 or later) was associated with lower FT4, whereas longer term use, was not (Fig. 5). Furthermore, a similar effect was indicated in two longitudinal nonrandomized studies exploring the impact of iodine supplement initiated early in pregnancy on thyroid function in mild to moderately ID populations by Moleti et al. (median UIC $\sim 60 \mu \mathrm{g} / \mathrm{L}$ in nonsupplemented women) $(21,47)$. Also, Rebagliato et al. reported increased risk of elevated TSH in pregnant women taking supplements containing $200 \mu \mathrm{g}$ iodine per day or more compared to $0-100 \mu \mathrm{g} /$ day (22). Contrary to this, no difference was seen in a recent RCT of iodine supplementation in pregnancy by Gowachirapant et al. in a mildly ID population (median UIC $131 \mu \mathrm{g} / \mathrm{L}$ at baseline) (23). Interestingly, using data from MoBa, we have previously reported that maternal iodine supplement use initiated in the first trimester was associated with more behavior problems in 3 year old children (3) and increased risk of ADHD diagnosis in 6-14 year old children (4). These associations could be explained by a temporary lower thyroid hormone production due to an acute higher iodine availability in this vulnerable window of neurodevelopment. Taken together, these results indicate that mild- to moderate ID should optimally be prevented before pregnancy since initiating supplement use in pregnancy might be too late and may have adverse effects on thyroid function.

Several nutrients and nonnutrients are known to affect thyroid function $(8,48)$. We were not able to detect effect modification on the association of iodine intake with thyroid function by either low iron or selenium status, smoking, or intake of cruciferous vegetables. Iron is essential for the activity of thyroid peroxidase in the production of thyroid hormones. Selenium is an integral part of selenoenzymes protecting the thyroid from excess $\mathrm{H}_{2} \mathrm{O}_{2}$ produced in thyroid hormone synthesis. Selenium is also incorporated in deiodinase enzymes crucial for regulation of thyroid hormone activity and thus action in target-tissues. Cruciferous vegetables and smoking both contribute with goitrogenic compounds inhibiting iodine uptake in the thyroid. In our study population, the prevalence of smoking, selenium deficiency, and the intake of cruciferous vegetables were all low limiting the ability to explore potential effect modifications. Therefore, we cannot conclude that such effects were not present as this study was probably underpowered to detect such interactions. Some studies have indicated that ID populations might be more vulnerable to thyroid disrupting substances (48), and that concurrent iron and/or selenium deficiency might have synergistic effects with ID on thyroid function (49).

\section{Strengths and limitations}

The present study has several limitations. Most importantly, the participants in this study had an insufficient iodine intake regardless of their reported food and supplement intake (i.e., median UIC $\geq 150 \mu \mathrm{g} / \mathrm{L}$, see Fig. 2 and Table 1). Thus, we might lack an iodine sufficient group for comparison. However, the cutoff of $150 \mu \mathrm{g} / \mathrm{L}$ for defining inadequacy may be too high. In the Nordic countries, the recommended iodine intake for pregnant women of $175 \mu \mathrm{g} / \mathrm{d}$ is only $60 \%$ the WHO recommendation of $250 \mu \mathrm{g} / \mathrm{d}$ (14), corresponding to a median UIC $\geq 105 \mu \mathrm{g} / \mathrm{L}$. Nevertheless, median UIC was below $100 \mu \mathrm{g} / \mathrm{L}$ even in the participants with the highest reported iodine intake from food (Fig. 2) as well as in current supplement users. Participants who reported use of iodinecontaining supplements all time periods from 6 months before pregnancy probably had adequate iodine status, but they were few $(n=138)$.

The cross-sectional design is a limitation preventing us to study potential intra-individual changes in thyroid function parameters by short-term iodine availability. In addition, the observational design means that residual confounding cannot be ruled out. This study included a selected sample of highly motivated mothers with offspring with low prevalence of language delay and no autism (Fig. 1). Increased risk of 
Table 3. The 2.5Th and 97.5th Percentiles of TSH, FT4, and FT3 by Measures of Iodine Status (Adjusted Models)

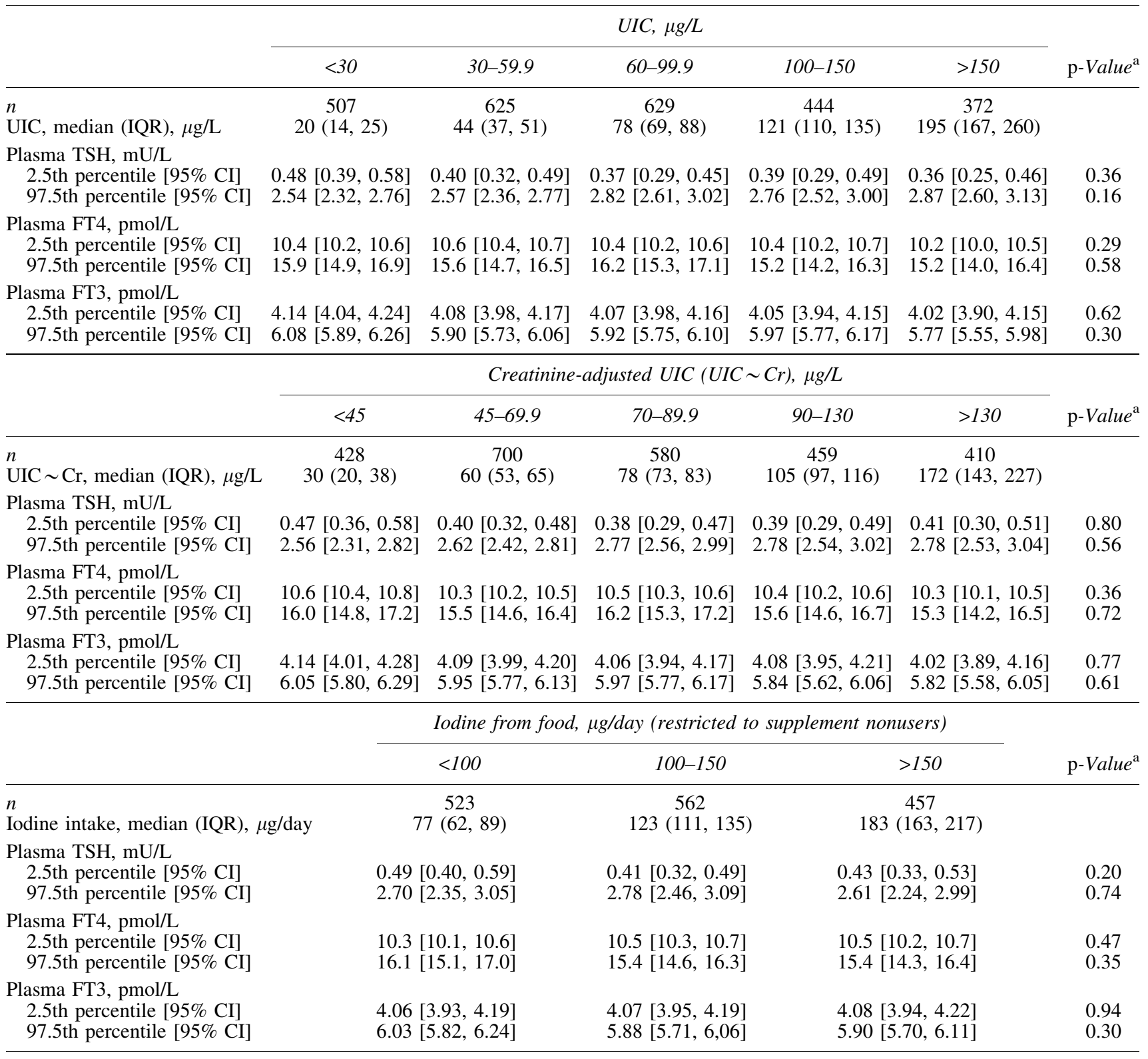

Data from adjusted models: patients with TPOAb positive (TPOAb $>4.11 \mathrm{IU} / \mathrm{mL}$ ) and current users of thyroid medication or thyroid disrupting medication, and women who have undergone in vitro fertilization were excluded.

Values are from quantile regression and adjusted for maternal age, prepregnancy BMI, gestational age at sampling, urinary creatinine (when UIC $\sim \mathrm{Cr}$ is the exposure), and energy intake (when iodine from food is the exposure). Results from crude models are reported in Supplementary Table S2.

${ }^{a} p$-Value from quantile regression.

language delay was previously documented in children of mothers with low iodine intake in $\mathrm{MoBa}$ (3), thus potentially, the prevalence of mothers with altered thyroid function due to ID might be lower in our study sample. Substantial measurement error of the exposure variables in measuring actual iodine intake probably also contributed to an attenuation of the associations. Although the total number of participants was large, there was limited power to study potential effect modifications by iron status, selenium status, smoking, and goitrogens in cruciferous vegetables.

Strengths of the study include the relatively large number of participants, the substantial variation in iodine intake, having two different measures of iodine intake (i.e., one for habitual intake and one for short-term intake) and the extensive data collection allowing us to adjust for many confounding factors.

\section{Clinical relevance and implications}

Overall, the findings in this study indicate that a habitual iodine intake below approximately $150 \mu \mathrm{g} /$ day is associated with changes in maternal thyroid hormone concentrations in pregnancy but not with significant differences in the reference range limits. Many studies have demonstrated that the 

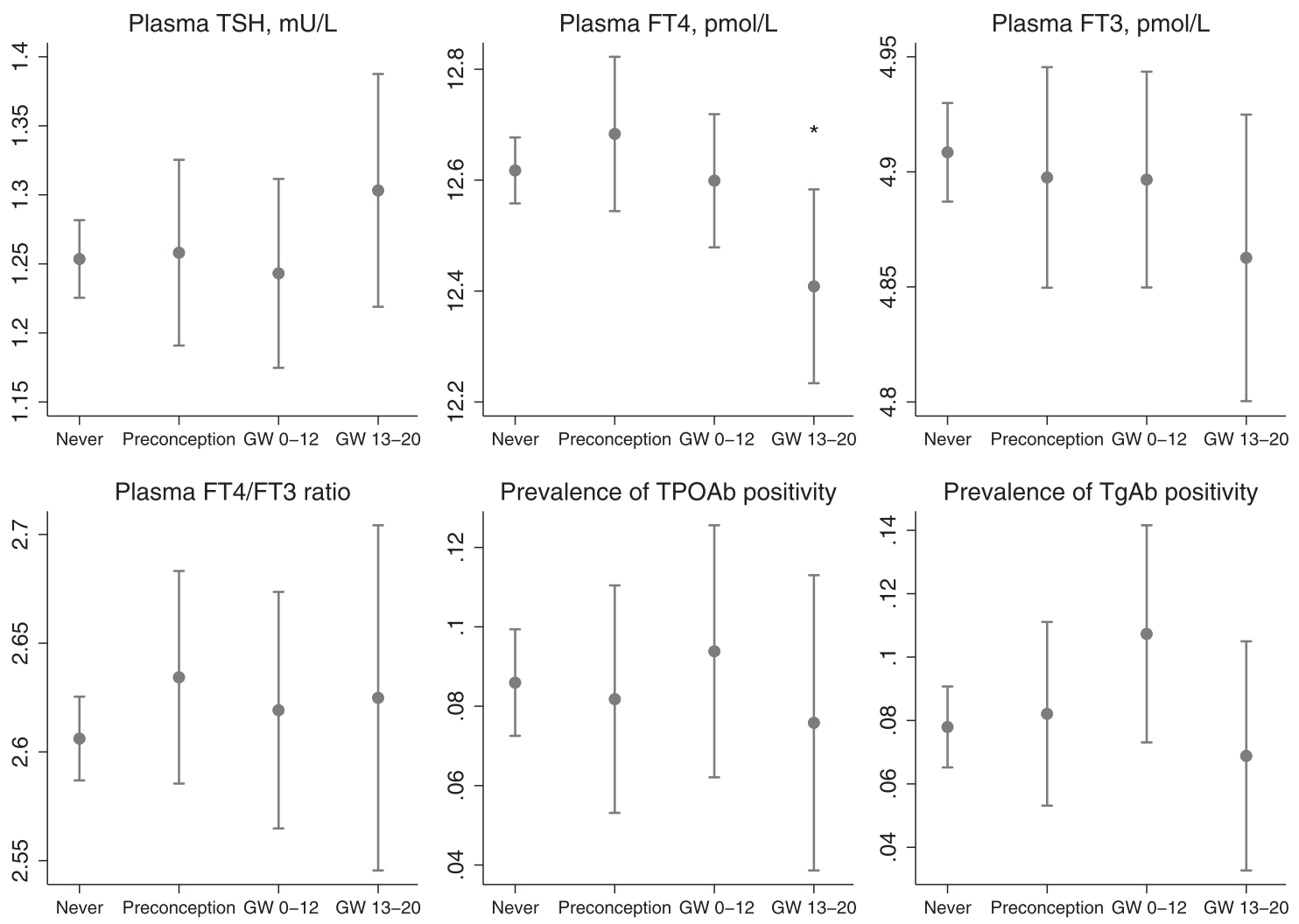

FIG. 5. Timing of initiation of iodine-containing supplements in pregnancy and measures of maternal thyroid function, adjusted models. Timing was categorized as: no reported supplement use (never; $n=1738$ ), first use in weeks $1-26$ before conception (preconception; $n=347$ ), first use during first trimester (GW 0-12; $n=323$ ), and first use during second trimester (GW 13-20; $n=194)$. Participants who reported use of iodine-containing supplements in the FFQ but did not report timing of use $(n=308)$ were not included in this analysis. For TSH, the geometric mean was modelled (by square root transformation), and for FT3 and FT4 the highest first percentile was excluded to obtain $\sim$ normal distribution. Crude models are included in Supplementary Fig. S10, and models excluding antibody positive women in Supplementary Fig. S9. * $p<0.05$. $\mathrm{GW}$, gestational week.

developing fetus might be vulnerable to maternal thyroid hormone supply, particularly in the first half of pregnancy (45). Only $30 \%$ of the MoBa women reached an iodine intake from food sources of $150 \mu \mathrm{g} / \mathrm{day}$, which is equivalent to the recommended intake for nonpregnant women.

Current international guidelines recommend that gestational thyroid function reference ranges are calculated in an iodine-sufficient population. To our knowledge, there are no studies that have shown that thyroid function reference ranges during pregnancy differ according to iodine status. Our results indicate that mild iodine deficiency is not a determinant of thyroid function reference ranges during pregnancy and that valid thyroid function reference ranges can be ascertained also in populations classified as being mildly to moderately iodine deficient. However, this finding needs to be confirmed in other studies before changing the guidelines.

We also found that initiating iodine supplement use in pregnancy in a population with mild to moderate ID diets was associated with lower FT4, which may be harmful for the developing child. Thus, this study provides further supporting evidence to the recommendation by the WHO that strategies to prevent iodine deficiency should target the whole population, and in particular all women of childbearing age, so that an adequate iodine status is secured well before conception. Today, there is a lack of evidence to support recommending iodine supplements for pregnant women in mild to moderately iodine deficient populations $(5,20)$.

\section{Conclusion}

All in all, the results indicate that mild to moderate iodine deficiency is associated with thyroid hormone levels in pregnancy. However, the changes were small, suggesting that normal reference ranges can be determined based on data also from mildly iodine deficient populations, but this must be confirmed in other studies. Introducing an iodine-containing supplement might temporarily inhibit thyroid hormone production and/or release, thus ID should ideally be prevented before conception.

\section{Acknowledgments}

We are grateful to all the participating families in Norway who take part in this on-going cohort study. The Norwegian Mother and Child Cohort Study is supported by the Norwegian Ministry of Health and the Ministry of Education and Research, National Institutes of Health/National Institute of Environmental Health Sciences (contract No. N01-ES-75558), National Institutes of Health/National Institute of Neurological Disorders and Stroke (grant Nos. UO1 NS 047537-01 and UO1 NS 047537-06A1). M.H.A. 
was supported by a four-year grant from the Norwegian Research Council (grant No. 241430) and the Norwegian dairy company TINE SA.

\section{Author Disclosure Statement}

M.H. Abel is employed by a Norwegian dairy company (TINE SA), and she participates in this project as an industrial PhD student financed partly by the dairy company and partly by The Research Council of Norway. This project is designed, owned, and administered by The Norwegian Institute of Public Health and analysis of the data follow from protocol. All results of analyses in the project are to be published regardless of the results. The dairy company supports the study to raise awareness on the importance of iodine and to gain more knowledge about the potential health effects of milk in the Norwegian diet. Apart from M.H.A., no one from the dairy company has been involved in the study, and in itself, the company had no direct influence on the analysis and interpretation of the results. All remaining authors declare no competing financial interests.

\section{References}

1. Bath SC, Steer CD, Golding J, Emmett P, Rayman MP 2013 Effect of inadequate iodine status in UK pregnant women on cognitive outcomes in their children: results from the Avon Longitudinal Study of Parents and Children (ALSPAC). Lancet 382:331-337.

2. Hynes KL, Otahal P, Burgess JR, Oddy WH, Hay I 2017 Reduced educational outcomes persist into adolescence following mild iodine deficiency in utero, despite adequacy in childhood: 15-Year follow-up of the Gestational Iodine Cohort investigating auditory processing speed and working memory. Nutrients 9:pii:E1354.

3. Abel MH, Caspersen IH, Meltzer HM, Haugen M, Brandlistuen RE, Aase H, Alexander J, Torheim LE, Brantsaeter AL 2017 Suboptimal maternal iodine intake is associated with impaired child neurodevelopment at 3 years of age in the Norwegian Mother and Child Cohort Study. J Nutr 147: 1314-1324.

4. Abel MH, Ystrom E, Caspersen IH, Meltzer HM, Aase H, Torheim LE, Askeland RB, Reichborn-Kjennerud T, Brantsaeter AL 2017 Maternal iodine intake and offspring attention-deficit/hyperactivity disorder: Results from a large prospective cohort study. Nutrients 9.

5. Pearce EN, Lazarus JH, Moreno-Reyes R, Zimmermann MB 2016 Consequences of iodine deficiency and excess in pregnant women: an overview of current knowns and unknowns. Am J Clin Nutr 104:918S-923S.

6. Redman K, Ruffman T, Fitzgerald P, Skeaff S 2016 Iodine deficiency and the brain: Effects and mechanisms. Crit Rev Food Sci Nutr 56:2695-2713.

7. Aburto N, Abudou M, Candeias V, Wu T 2014 Effect and safety of salt iodization to prevent iodine deficiency disorders: a systematic review with meta-analyses. In: World Health Organization [WHO] eLibrary of Evidence for Nutrition Actions (eLENA). World Health Organization, Geneva.

8. O'Kane SM, Mulhern MS, Pourshahidi LK, Strain JJ, Yeates AJ 2018 Micronutrients, iodine status and concentrations of thyroid hormones: a systematic review. Nutr Rev 76:418-431.

9. Roman GC 2007 Autism: transient in utero hypothyroxinemia related to maternal flavonoid ingestion during pregnancy and to other environmental antithyroid agents. J Neurol Sci 262:15-26.

10. Shi X, Han C, Li C, Mao J, Wang W, Xie X, Li C, Xu B, Meng T, Du J, Zhang S, Gao Z, Zhang X, Fan C, Shan Z, Teng W 2015 Optimal and safe upper limits of iodine intake for early pregnancy in iodine-sufficient regions: a cross-sectional study of 7190 pregnant women in China. J Clin Endocrinol Metab 100:1630-1638.

11. Laurberg P, Cerqueira C, Ovesen L, Rasmussen LB, Perrild $\mathrm{H}$, Andersen S, Pedersen IB, Carle A 2010 Iodine intake as a determinant of thyroid disorders in populations. Best Pract Res Clin Endocrinol Metab 24:13-27.

12. Andersson M, de Benoist B, Delange F, Zupan J 2007 Prevention and control of iodine deficiency in pregnant and lactating women and in children less than 2-years-old: conclusions and recommendations of the technical consultation. Public Health Nutr 10:1606-1611.

13. EFSA NDA Panel (EFSA Panel on Dietetic Products Nutrition and Allergies) 2014 Scientific opinion on dietary reference values for iodine. EFSA J 12:3660.

14. NNR12 Project Group 2014 Iodine in Nordic Nutrition Recommendations 2012, Integrating Nutrition and Physical Activity. Fifth edition. Nordic Council of Ministers, Copenhagen, pp 583-590.

15. Alexander EK, Pearce EN, Brent GA, Brown RS, Chen H, Dosiou C, Grobman WA, Laurberg P, Lazarus JH, Mandel SJ, Peeters RP, Sullivan S 20172017 Guidelines of the American Thyroid Association for the diagnosis and management of thyroid disease during pregnancy and the postpartum. Thyroid 27:315-389.

16. Food and Nutrition Board, Institute of Medicine 2001 Iodine dietary reference intakes for vitamin A, vitamin $\mathrm{K}$, arsenic, boron, chromium, copper, iodine, iron, manganese, molybdenum, nickel, silicon, vanadium, and zinc. National Academies Press, Washington (DC), 258-289.

17. Korevaar TIM, Medici M, Visser TJ, Peeters RP 2017 Thyroid disease in pregnancy: new insights in diagnosis and clinical management. Nat Rev Endocrinol 13:610-622.

18. World Health Organization, United Nations Children's Fund, International Council for Control of Iodine Deficiency Disorders 2007 Assessment of Iodine Deficiency Disorders and Monitoring Their Elimination: A Guide for Programme Managers. Third edition. WHO, Geneva, Switzerland.

19. Becker DV, Braverman LE, Delange F, Dunn JT, Franklyn JA, Hollowell JG, Lamm SH, Mitchell ML, Pearce E, Robbins J, Rovet JF 2006 Iodine supplementation for pregnancy and lactation-United States and Canada: recommendations of the American Thyroid Association. Thyroid 16:949-951.

20. Harding KB, Pena-Rosas JP, Webster AC, Yap CM, Payne BA, Ota E, De-Regil LM 2017 Iodine supplementation for women during the preconception, pregnancy and postpartum period. Cochrane Database Syst Rev 3:CD011761.

21. Moleti M, Di Bella B, Giorgianni G, Mancuso A, De Vivo A, Alibrandi A, Trimarchi F, Vermiglio F 2011 Maternal thyroid function in different conditions of iodine nutrition in pregnant women exposed to mild-moderate iodine deficiency: an observational study. Clin Endocrinol (Oxf) 74:762-768.

22. Rebagliato M, Murcia M, Espada M, Alvarez-Pedrerol M, Bolumar F, Vioque J, Basterrechea M, Blarduni E, Ramon R, Guxens M, Foradada CM, Ballester F, Ibarluzea J, Sunyer J 2010 Iodine intake and maternal thyroid function during pregnancy. Epidemiology 21:62-69.

23. Gowachirapant S, Jaiswal N, Melse-Boonstra A, Galetti V, Stinca S, Mackenzie I, Thomas S, Thomas T, Winichagoon 
P, Srinivasan K, Zimmermann MB 2017 Effect of iodine supplementation in pregnant women on child neurodevelopment: a randomised, double-blind, placebo-controlled trial. Lancet Diabetes Endocrinol 5:853-863.

24. Taylor PN, Okosieme OE, Dayan CM, Lazarus JH 2014 Therapy of endocrine disease: Impact of iodine supplementation in mild-to-moderate iodine deficiency: systematic review and meta-analysis. Eur J Endocrinol 170:R1-R15.

25. Zhou SJ, Anderson AJ, Gibson RA, Makrides M 2013 Effect of iodine supplementation in pregnancy on child development and other clinical outcomes: a systematic review of randomized controlled trials. Am J Clin Nutr 98:1241-1254.

26. Magnus P, Birke C, Vejrup K, Haugan A, Alsaker E, Daltveit AK, Handal M, Haugen M, Hoiseth G, Knudsen GP, Paltiel L, Schreuder P, Tambs K, Vold L, Stoltenberg C 2016 Cohort profile update: The Norwegian Mother and Child Cohort Study (MoBa). Int J Epidemiol 45:382-388.

27. Irgens LM 2000 The Medical Birth Registry of Norway. Epidemiological research and surveillance throughout 30 years. Acta Obstet Gynecol Scand 79:435-439.

28. Meltzer HM, Brantsaeter AL, Ydersbond TA, Alexander J, Haugen M 2008 Methodological challenges when monitoring the diet of pregnant women in a large study: experiences from the Norwegian Mother and Child Cohort Study (MoBa). Matern Child Nutr 4:14-27.

29. Norwegian Institute of Public Health website, MoBa Food Frequency Questionnaire (English translation). Available at: www.webcitation.org/6u5JMPcZg (accessed October 9, 2017).

30. Dahl L, Johansson L, Julshamn K, Meltzer HM 2004 The iodine content of Norwegian foods and diets. Public Health Nutr 7:569-576.

31. Dahl L, Opsahl JA, Meltzer HM, Julshamn K 2003 Iodine concentration in Norwegian milk and dairy products. Br J Nutr 90:679-685.

32. Haugen M, Brantsaeter AL, Alexander J, Meltzer HM 2008 Dietary supplements contribute substantially to the total nutrient intake in pregnant Norwegian women. Ann Nutr Metab 52:272-280.

33. Brantsaeter AL, Haugen M, Alexander J, Meltzer HM 2008 Validity of a new food frequency questionnaire for pregnant women in the Norwegian Mother and Child Cohort Study (MoBa). MaternChild Nutr 4:28-43.

34. Brantsaeter AL, Abel MH, Haugen M, Meltzer HM 2013 Risk of suboptimal iodine intake in pregnant Norwegian women. Nutrients 5:424-440.

35. Brantsaeter AL, Haugen M, Hagve TA, Aksnes L, Rasmussen SE, Julshamn K, Alexander J, Meltzer HM 2007 Self-reported dietary supplement use is confirmed by biological markers in the Norwegian Mother and Child Cohort Study (MoBa). Ann Nutr Metab 51:146-154.

36. Brantsaeter AL, Haugen M, Julshamn K, Alexander J, Meltzer HM 2009 Evaluation of urinary iodine excretion as a biomarker for intake of milk and dairy products in pregnant women in the Norwegian Mother and Child Cohort Study (MoBa). Eur J Clin Nutr 63:347-354.

37. Ronningen KS, Paltiel L, Meltzer HM, Nordhagen R, Lie KK, Hovengen R, Haugen M, Nystad W, Magnus P, Hoppin JA 2006 The biobank of the Norwegian Mother and Child Cohort Study: a resource for the next 100 years. Eur J Epidemiol 21:619-625.

38. Willett WC, Howe GR, Kushi LH 1997 Adjustment for total energy intake in epidemiologic studies. Am J Clin Nutr 65:1220S-1228S; discussion 1229S-1231S.
39. Murcia M, Espada M, Julvez J, Llop S, Lopez-Espinosa MJ, Vioque J, Basterrechea M, Riano I, Gonzalez L, AlvarezPedrerol M, Tardon A, Ibarluzea J, Rebagliato M 2018 Iodine intake from supplements and diet during pregnancy and child cognitive and motor development: the INMA Mother and Child Cohort Study. J Epidemiol Community Health 72:216-222.

40. Korevaar TIM, Pop VJ, Chaker L, Goddijn M, de Rijke YB, Bisschop PH, Broeren MA, Jaddoe VWV, Medici M, Visser TJ, Steegers EAP, Vrijkotte TG, Peeters RP 2018 Dosedependency and a functional cut-off for TPO-antibody positivity during pregnancy. J Clin Endocrinol Metab 103: 778-789.

41. Buis M 2013 Postrcspline: Stata module containing postestimation commands for models using a restricted cubic spline. Statistical Software Components S456928, Boston College Department of Economics, revised 13 Dec 2013. Available at: https://econpapers.repec.org/software/bocbo code/s456928.htm (accessed September 8, 2017).

42. Mridha MK, Matias SL, Paul RR, Hussain S, Khan MSA, Siddiqui Z, Ullah B, Sarker M, Hossain M, Young RT, Arnold CD, Dewey KG 2017 Daily consumption of lipidbased nutrient supplements containing 250 mug iodine does not increase urinary iodine concentrations in pregnant and postpartum women in Bangladesh. J Nutr 147:1586-1592.

43. de Escobar GM, Obregon MJ, del Rey FE 2007 Iodine deficiency and brain development in the first half of pregnancy. Public Health Nutr 10:1554-1570.

44. Obregon MJ, Escobar del Rey F, Morreale de Escobar G 2005 The effects of iodine deficiency on thyroid hormone deiodination. Thyroid 15:917-929.

45. Velasco I, Bath SC, Rayman MP 2018 Iodine as essential nutrient during the first 1000 days of life. Nutrients 10:E290.

46. Levie D, Korevaar TIM, Bath SC, Dalmau-Bueno A, Murcia M, Espada M, Dineva M, Ibarluzea JM, Sunyer J, Tiemeier H, Rebagliato M, Rayman MP, Peeters RP, Guxens M 2018 Thyroid function in early pregnancy, child IQ, and autistic traits: a meta-analysis of individualparticipant data. J Clin Endocrinol Metab 103:2967-2979.

47. Moleti M, Lo Presti VP, Campolo MC, Mattina F, Galletti M, Mandolfino M, Violi MA, Giorgianni G, De Domenico D, Trimarchi F, Vermiglio F 2008 Iodine prophylaxis using iodized salt and risk of maternal thyroid failure in conditions of mild iodine deficiency. J Clin Endocrinol Metab 93:2616-2621.

48. Mughal BB, Fini JB, Demeneix BA 2018 Thyroid-disrupting chemicals and brain development: an update. Endocr Connect 7:R160-R186.

49. Hess SY 2010 The impact of common micronutrient deficiencies on iodine and thyroid metabolism: the evidence from human studies. Best Pract Res Clin Endocrinol Metab 24:117-132.

Address correspondence to:

Anne Lise Brantsater, PhD Department of Environmental Exposure and Epidemiology

Division of Infection Control and Environmental Health Norwegian Institute of Public Health P.O. Box 4404 Oslo NO-0403 Norway

E-mail: annelise.brantsaeter@fhi.no 